\title{
Anabases
}

ANABASES Traditions et réceptions de l'Antiquité

19 | 2014

Varia

\section{Pandora, ou le bonheur originel selon Voltaire}

\section{Béatrice Ferrier}

\section{OpenEdition}

Journals

Édition électronique

URL : http://journals.openedition.org/anabases/4706

DOI : 10.4000/anabases.4706

ISSN : 2256-9421

\section{Éditeur}

E.R.A.S.M.E.

\section{Édition imprimée}

Date de publication : 1 avril 2014

Pagination : 313-325

ISSN : 1774-4296

\section{Référence électronique}

Béatrice Ferrier, «Pandora, ou le bonheur originel selon Voltaire », Anabases [En ligne], 19 | 2014, mis en ligne le 01 avril 2017, consulté le 20 octobre 2019. URL : http://journals.openedition.org/anabases/ 4706 : DOI : 10.4000/anabases.4706

Ce document a été généré automatiquement le 20 octobre 2019

(c) Anabases 


\title{
Pandora, ou le bonheur originel selon Voltaire
}

\author{
Béatrice Ferrier
}

1 Tandis que la Théogonie narre les aventures de Prométhée, les Travaux et les Jours, poème moral qu'Hésiode adresse à son frère pour l'encourager à suivre la voie du Bien et du travail, s'attardent sur la fable de Pandore. Désormais, un nom est attribué à cette première femme dotée de toutes les séductions mais porteuse des maux de l'humanité, cette créature des dieux par laquelle Zeus entend se venger du voleur de feu : Pandora, "présent de tous ", car « ce sont tous les habitants de l'olympe qui, avec ce présent, font présent du malheur aux hommes qui mangent le pain ${ }^{1} »$. L'onomastique renforce ainsi l'intention didactique d'Hésiode, ce que confirment les identités signifiantes des deux frères construites sur une opposition de caractère déterminante pour la conduite du récit : le prévoyant Prométhée et l'irréfléchi Épiméthée ${ }^{2}$.

2 Cette dimension didactique, Voltaire l'apprécie tout particulièrement chez Hésiode ${ }^{3}$. Il présente d'ailleurs son livret de Pandore, entrepris dès 1739, comme "un opéra philosophique qui devrait être joué devant Bayle et Diderot $^{4}$ » et qui, à la suite de Samson, remet en cause l'autorité de l'Ancien Testament par une interprétation nouvelle $\mathrm{du}$ "péché originel $^{5}$ ». Toutefois, si Voltaire invite le cercle privé de ses correspondants à une lecture allégorique, il ne s'agit ni de dispenser le message d'Hésiode - il ne convient pas de faire partie des imitatores ${ }^{6}-$, ni de suivre les principes que Madame Dacier avait tenté de réhabiliter en ouvrant la seconde Querelle des Anciens et des Modernes par sa traduction de l'Iliade en 1711. C'est en analysant la manière dont est appréhendée la lecture allégorique que nous interrogerons les connotations nouvelles, fruits de déplacements, de travestissement, d'inversion ou d'innovation, que revêtent le nom, et par conséquent, le mythe de Pandora dans l'imaginaire voltairien.

3 Alors même que les dons justifient l'étymologie de Pandora, le livret de Voltaire tend à les estomper - les premiers vers nomment le personnage sans référer à cette qualité7. Pandore apparaît d'emblée comme un personnage littéraire. Contrairement au texte source, elle n'a pas été façonnée par Héphaïstos mais par le Titan lui-même, une 
résurgence du mythe de Pygmalion entérinée par la tradition. Dans la suite de sa récente lecture de la Mythologie de l'abbé Banier, Voltaire a également pu opérer un déplacement depuis la figure de Prométhée sculpteur, qui « forma le corps de l'homme avec de la boue détrempée ${ }^{8}$ ».

4 À l'évidence, un tel choix permet de réduire considérablement la part du divin. Les célèbres présents, longuement détaillés par Hésiode, sont à peine évoqués à l'acte IV du livret. Ils se limitent aux maux contenus dans la boîte, matérialisés sur scène par les furies et les démons9. En ce sens, Pandore n'est plus le "présent de tous ", dans l'acception antiphrastique de faux présent, de piège, puisque sa belle enveloppe charnelle ne cache ni une irrémédiable curiosité, ni un talent inné de la dissimulation et de la manipulation. Le personnage féminin est ainsi distinct du Mal incarné par le personnel allégorique traditionnel de l'Opéra. Le motif du don, du présent maléfique, est alors l'occasion d'un grand spectacle qui encadre la pièce : il est annoncé par Némésis au premier acte et confirmé au cinquième. Le nom de Pandora s'écarte ainsi du sens littéral grec, et de son sens en contexte, oxymorique, de beau Mal, de «bestialité » cachée sous « l'éclat du divin ${ }^{10}$ ».

5 Pourtant le motif du don n'est pas absent du livret de Voltaire qui le décline différemment, par déplacements successifs, pour réorienter la fable.

6 Le « don " y apparaît sous un angle plus abstrait et métaphysique à travers les échanges entre les dieux et les hommes, justifiant dans le mythe leur séparation. Il alterne avec son antonyme, le rapt, selon une structure duelle ${ }^{11}$ : Prométhée vole le feu, Jupiter enlève Pandore. Ainsi s'expliquent les très attendues scènes qui revêtent une portée allégorique singulière en matérialisant les liens entre le Ciel et la Terre jusqu'à leur " divorce éternel ${ }^{12}$ ». La scénographie spectaculaire de Pandore envisagée présente les caractéristiques d'un opéra traditionnel à sujet mythologique, susceptible de corriger l'échec cuisant de Samson. Toutefois, de même que la scène des miracles de l'opéra biblique reposait sur l'artifice des machines ${ }^{13}$, de même la machine de gloire sans cesse en mouvement manifeste les relations tumultueuses entre l'olympe et la Terre. L'une des versions manuscrites interrompt précisément les envols à l'acte $\mathrm{V}$ au moment où la communication entre les deux mondes est définitivement rompue, le théâtre ménageant un simple changement à vue du décor avec l'apparition du "palais de l'amour ${ }^{14} »$.

7 En effet, le mythe de Pandore n'est autre que la version grecque du péché originel, selon la lecture comparatiste qu'affectionne Voltaire pour ôter toute autorité à la Bible $^{15}$, assimilée aux autres "fables allégoriques ${ }^{16}$ » des origines: Prométhée et Pandore équivalent à Adam et Ève. La disparition du personnage d'Épiméthée donne à voir le couple originel dans le jardin d'Éden symbolisé par les hymnes à la nature et par les décors de " campagne éclairée et riante » ou de " bocage ${ }^{17}$ ». Le passage de la Genèse que Voltaire entend ainsi parodier est celui de la chute de l'homme condamné à quitter le monde divin pour avoir goûté le fruit défendu : le piège tendu par Némésis rappelle la séduction perfide du serpent à l'égard de "Pandore-Ève ${ }^{18}$ " pour qu'elle ouvre la fatale boîte ou croque la pomme. Cette lecture allégorique du mythe de Pandore a cours depuis l'Antiquité ${ }^{19}$ en raison de l'analogie entre les deux créatures féminines, conçues par la main divine et responsables des souffrances humaines.

8 Néanmoins, le livret de Voltaire se distingue du Paradis perdu ${ }^{20}$ et des reprises dramatiques contemporaines en représentant un Prométhée en «révolte ${ }^{21}$ » contre Jupiter. Il en va de même pour la fidèle Pandore qui refuse l'offre d'immortalité de 
Jupiter et revendique son retour sur terre, en une scène d'affrontement que prolonge et achève le combat des Titans ${ }^{22}$. C'est contre l'injustice divine que s'insurge la première femme, relayant la lutte de Prométhée due à la seule jalousie de Jupiter (sans mention de leur rivalité antérieure ${ }^{23}$ ), qui refuse la vie au « divin ouvrage ${ }^{24}$ ». La responsabilité du sort des hommes repose donc entièrement sur la tyrannie d'un dieu jaloux et vengeur, à l'instar de Yahvé contraignant Samson à accomplir son destin en renversant le temple au lieu de conclure la paix avec les Philistins. Les analogies se retrouvent aussi dans la construction du personnage féminin dépourvu de perfidie, trait habituellement dévolu aux prêtres dans l'opéra biblique, aux dieux dans l'opéra mythologique.

9 Cette lecture allégorique inverse les intentions de légitimation de la Bible que l'approche comparatiste des Anciens défendait initialement ${ }^{25}$. Mais Voltaire ne jette pas seulement le discrédit sur un texte dont il dénie toute vérité révélée, il fait de Pandore la représentation de la lutte de l'homme innocent persécuté contre l'injustice divine et, au-delà, contre le jansénisme. C'est pourquoi il recommande de conserver les vers « $\hat{O}$ Jupiter, ô fureurs inhumaines! / Éternel persécuteur / De l'infortune créateur, etc. », avec leur coloration « très janséniste ${ }^{26}$ ».

10 Pandore revêt donc un sens polémique au-delà du sens métaphysique. Jouant des conventions de l'Opéra, Voltaire revendique l'innocence de l'homme en effaçant l'imprudence d'Épiméthée et les défauts d'Ève ou de Pandore. Cette dernière incarne le rôle que lui assigne la tradition en se laissant abuser par Némésis, travestie en messager digne de confiance ${ }^{27}$. Elle agit par crainte de perdre son amant. Le librettiste accroît par ailleurs le sentiment d'injustice en renforçant la valeur morale de Prométhée, lequel refuse l'aide des Enfers ${ }^{28}$ et court aider les Titans punis par le Destin plutôt que de goûter son bonheur auprès de Pandore. Or cette mise en accusation des dieux dans un système manichéen, qui justifie la séparation des deux mondes, se manifeste dans le personnage de Jupiter qui, indifféremment, « commande à l'olympe, à la terre, aux enfers $^{29}{ }^{2}$. C'est ainsi que Némésis, renvoyée aux Enfers par Prométhée, se trouve investie de sa mission maléfique par le dieu de l'olympe lui-même ${ }^{30}$, dont les actes semblent orchestrés par le Destin ${ }^{31}$, un personnage allégorique fréquent à l'Opéra mais peu innocent sous la plume du philosophe compte tenu de la place de la prédestination chez les jansénistes.

11 Par conséquent, la portée métaphysique du don invite à une lecture philosophique bien éloignée de l'interprétation que les Pères de l'Église proposaient du péché originel, Voltaire récusant la damnation de l'homme: "Celui qui inventa la fable de Pandore avait plus d'esprit que Saint Augustin, et était beaucoup plus raisonnable. Il ne damne point les enfants de notre mère Pandore; il se contente de leur donner la fièvre, la goutte, la gravelle par héritage ${ }^{32}$.» Tout comme il utilise la Bible à des fins contraires, Voltaire se sert de la fable d'Hésiode pour s'engager dans la polémique: Hésiode n'encourage pas à la révolte contre les dieux mais à la soumission.

12 Au-delà de la volonté de dénoncer l'interprétation judéo-chrétienne du péché originel, le philosophe défend une certaine conception de la nature humaine et de sa propension au bonheur. Celle-ci repose sur une autre forme de don, d'ordre symbolique: Prométhée, « un flambeau à la main ${ }^{33}$ », anime le corps de Pandore.

13 Ce don immatériel passe par le feu, un «feu sacré34 " qui chez Voltaire, dans la continuité d'Hésiode ${ }^{35}$, métaphorise la vie mais également l'amour, présenté comme sa condition même : «À sa voix obéis toujours; / Lève-toi, l'amour te donne / La vie, un 
cœur et de beaux jours ${ }^{36}$.» Le rythme ternaire du vers final établit ainsi l'équivalence entre le souffle vital et la capacité d'aimer et d'être heureux. L'impératif, en écho à la parole christique, prend le contre-pied d'une religion fondée sur la culpabilité de l'homme pour prôner le droit de celui-ci au bonheur. Quelques années plus tôt, en 1734, Voltaire ne récusait-il pas dans ses Lettres philosophiques « l'abîme du péché originel ${ }^{37}$ »? En ce sens, le seul don sur lequel repose la Pandore de Voltaire est celui de l'amour à l'origine de la «douce espérance ${ }^{38}$ » qu'abrite le cœur des hommes, alors que le péché originel, celui de la connaissance, a été assimilé par la tradition au péché de la chair. Ce don fonde l'essence de l'humanité et engendre la jalousie de Jupiter.

Le finale de l'opéra consacre la victoire de l'amour symbolisée par l'apparition de son palais lors d'un changement à vue conforme au grand spectacle attendu. Aux ténèbres, dramatisant l'ouverture de la " boîte odieuse ", " ce don détesté39 ", succède selon toute probabilité la lumière - aucune didascalie ne l'indique explicitement - au moment où l'amour descend sur terre, un amour porteur d'espoir. En témoigne ce distique réparti entre les voix de Pandore et de l'Amour, « descendant du ciel » : « Tous les maux sont venus de la triste Pandore. / Tous les biens sont à vous, l'amour vous reste encore ${ }^{40}$." L'anaphore antithétique entérine la transformation du malheur en bonheur. C'est l'amour qui rend possible le bonheur terrestre en dépit des difficultés de la condition humaine, ce bonheur qui appartenait dans Samson au temps révolu des origines et d'Adonis ${ }^{41}$. Voltaire dispense cette fois un message optimiste dans Pandore en réagençant de nombreuses scènes du livret précédent. Tandis que le temple de Vénus qui abritait un duo d'amour provoquait la colère divine, plongeant le théâtre dans une pénombre et déclenchant une scène épique, l'inverse se produit dans Pandore: le temple de l'amour vient sauver les hommes d'un sort funeste engendré par la vengeance des dieux.

Cette célébration finale de l'amour, plus conforme à la tradition de l'Opéra, aurait pu correspondre aux fêtes données lors des mariages royaux ${ }^{42}$. Toutefois, la hardiesse de Voltaire consiste à relire, sous le voile apparent de la fable païenne, l'épisode de la Genèse en faisant basculer le sort de l'homme, un sort désormais enviable et heureux après son départ du jardin d'Éden. La première femme n'est plus une dangereuse pécheresse mais celle qui sauve l'humanité, qui lui donne accès à la connaissance et au bonheur en favorisant la rupture avec le divin. Est ainsi remise en question la religion, au sens étymologique de « religare » (ce qui relie l'homme à Dieu), au nom de l'amour qui relie entre eux les êtres.

16 Pandore renverse la damnation ${ }^{43}$ en élection grâce au pouvoir de l'amour, synonyme de la vie et de l'âme ${ }^{44}$. C'est pourquoi le présent du feu, de la lumière, vie et amour à la fois, devient le don suprême, un présent « cent fois préférable à ce présent cruel ${ }^{45}$ » qu'est l'immortalité des dieux, à jamais perdue par Adam. L'allusion à l'immortalité de l'âme et sa remise en cause transparaissent sous la plume du philosophe qui conteste toute forme de révélation de la part de Moïse ${ }^{46}$. Pandore choisit donc l'âme mortelle, associée à l'amour nommé «âme de mon âme », Prométhée apparaissant comme le "maître de [s]on âme ${ }^{47}$ ». Voltaire reprend ici la métaphore classique de la flamme amoureuse pour l'assimiler autant à la flamme de la vie qu'au symbole de l'âme, dans la lignée de la philosophie antique où Pandore était, précisément, associée à l'âme ${ }^{48}$.

17 Ce n'est pas la première fois que Voltaire célèbre l'amour dans un livret d'opéra. Depuis Tanis et Zélide, en passant par Samson, qui sont autant de textes relais, le même message est diffusé, avec des variantes (attaques contre les religions ou dénonciation de dieux 
injustes) et à partir de mythologies différentes (égyptienne, judaïque ou grecque) jugées équivalentes. Il s'agit de célébrer la nature des origines et le bonheur terrestre autour de la force de l'amour, autour d'un symbole placé par les Pères de l'Église sous le sceau du péché originel. C'est l'amour que Voltaire retient dans la fable d'Hésiode ${ }^{49}$ et dans le poème de Milton $^{50}$. Il entreprend d'ailleurs Pandore après avoir commencé à travailler, sous l'impulsion des idées de Pope, au Discours en vers sur l'homme dont les deux premières Épitres sur le bonheur, publiées anonymement en mars 1738, échafaudent un système moral sur la recherche du bonheur ${ }^{51}$.

Par conséquent, Voltaire s'écarte du sens littéral conféré par Hésiode à Pandora pour l'enrichir de sens nouveaux en déclinant le motif du «don» selon deux lectures allégoriques complémentaires. La première détourne les principes du comparatisme, au cœur des débats sur le merveilleux chrétien tels que Madame Dacier les avait réactivés ou avait tenté de le faire. Il ne s'agit plus de légitimer la Bible par le biais de la mythologie mais de l'utiliser en vue d'une interprétation de la Genèse contraire à celles des Pères de l'Église, donnant à lire le péché originel comme la source même du bonheur humain. Du "présent de tous ", maléfique, Pandora est investie d'un sens opposé puisqu'elle devient l'incarnation du Bien par la vertu intrinsèque de l'amour. La première femme octroie donc à tous les hommes un présent inestimable, celui de la vie - Ève, en hébreu, signifiant la vie - mais aussi et surtout celui du bonheur universel, le nom de Pandora se transformant en "présent pour tous ${ }^{52}$ ". Au-delà de la relecture provocatrice et polémique du péché originel, c'est donc une nouvelle interprétation allégorique, de caractère philosophique, que propose Voltaire. La création de la première femme ne correspond plus à la chute de l'homme mais à l'ascension de celuici vers sa propre destinée, libérée du joug d'un Dieu vengeur. Cette délivrance n'est pas, selon Voltaire, la condition du malheur des hommes mais au contraire de leur accès au paradis terrestre éclairé par le Dieu Amour, assimilé à la Nature ${ }^{53}$, dans le sillage de Lucrèce qui voyait en Vénus «l'âme de l'univers ${ }^{54}$ ». Pandora devient le " présent pour tous les hommes » de la liberté et du bonheur, celle qui révèle à l'homme la toutepuissance de la Nature, de l'Amour, de la foi en un ordre naturel gouverné par une puissance bienfaisante, instaurant une forme nouvelle de religion, non plus la religion révélée qui divise les hommes, mais une religion qui réactive son sens étymologique en les reliant. Pandora révèle alors, dans la mythologie voltairienne, le bonheur originel auquel tout homme peut prétendre.

\section{NOTES}

1. Les Travaux et les Jours, trad. P. Mazon, Paris, Les Belles Lettres, CUF, 1928, v. 80-82.

2. Cf. R. TROUSSON, Le thème de Prométhée dans la littérature européenne, Genève, Droz, 1964, p. 32-40.

3. Cf. M. MAT-HASQUIN, Voltaire et l'Antiquité grecque, Oxford, Voltaire Foundation, 1981, p. 51-78.

4. V. à Laborde, 4 novembre 1765, D12966 - toutes nos références à la Correspondance de Voltaire sont empruntées à l'édition de Th. Besterman (Correspondence and Related Documents), Genève, Institut et musée Voltaire, puis Oxford, Voltaire Foundation, 1968-1978, 53 tomes. 
5. Selon le mot de Voltaire (lettre à Laborde précédemment citée), qui venait d'opérer dans le même sens, à partir d'un sujet biblique, dans le livret de Samson composé pour Rameau entre 1733 et 1736.

6. Référence à Horace (Épîtres, I, 19, v. 19 : Ô imitatores servum pecus...), que Voltaire invoque pour justifier son souhait de composer avec Samson un «nouveau genre d'opéra » (V. à Thieriot, 10 février 1736, D1008).

7. Vers 3 de Pandore, éd. R. Trousson, in Euvres complètes de Voltaire, Writing for music 1720-1740, Oxford, Voltaire Foundation, 2008, vol. 18 C, p. 363.

8. La Mythologie et les Fables expliquées par l'histoire, t. II, Paris, Briasson, 1738, p. 118. L'abbé Banier remarque d'ailleurs que parmi les diverses représentations antiques de Prométhée «inventant » l'homme, certaines ont d'évidentes affinités avec le mythe de Psyché (p. 121-122).

9. Matérialisation au demeurant fréquente dans les représentations de l'époque - I. Galleron cite Saint-Foix, Lesage, d'Orneval, ou encore le Père Brumoy (« La boîte de Pandore dans la caverne d'Alcandre: variations dramatiques sur un thème à la mode dans la première moitié du XviII siècle ", Séries et variations. Études littéraires offertes à Sylvain Menant, Paris, PUPS, 2010, p. 451-462).

10. J.-P. VERnANT, Mythe et Société en Grèce ancienne, Paris, La Découverte, 2004 [1 ${ }^{\text {re }}$ éd. : 1974], p. 192-193.

11. Cf. J.-P. Vernant, Mythe et Société..., p. 178-187, et D. SAintillan, «Du festin à l'échange : les grâces de Pandore ", in Le Métier du mythe, Lectures d'Hésiode, éd. par Fabienne Blaise, Pierre Judet de La Combe et Philippe Rousseau, Lille, P.U. de Septentrion, 1996, p. 315-348.

12. Pandore, acte IV, v. 73, p. 400 - la structure externe de Pandore se limite à un découpage par actes, le premier comptant néanmoins deux scènes; nous renvoyons donc ici aux numéros de vers et aux pages de l'édition de référence.

13. Cf. B. FERRIER, «Le Samson de Voltaire : un "nouveau genre d'opéra" ", in Cahiers Voltaire, 2008, $\mathrm{n}^{\circ}$ 7, p. 7-21.

14. Pandore, acte V, p. 414, note 148 a.

15. «Le premier Zoroastre avait aussi placé un homme et sa femme dans le paradis terrestre. Le premier homme était Micha, et la première femme Mishana. Chez Sanconiaton ce sont d'autres noms. Chez les brahmanes, c'est Adimo et Procriti. Chez les Grecs, c'est Prométhée et Pandore ; mais des siècles entiers de philosophes ne reconnurent pas plus un premier homme qu'un premier arbre. Chaque nation fit son système, et toutes avaient besoin de la révélation de Dieu même pour connaître ces choses sur lesquelles on dispute encore, et qu'il n'est pas donné à l'homme de connaître. » (art. " Genèse » de La Bible enfin expliquée par plusieurs aumôniers de S. M. L. R. D. P., t. I, Genève, [s. n.], 1776, p. 9, note 14).

16. L'Examen important de Milord Bolingbroke, chap. VI (« De la Genèse »), Londres, [s. n.], 1776 [1 ${ }^{\text {re }}$ éd. : 1766], p. 29.

17. Pandore, respectivement acte I, sc. 2, v. 80-81, p. 370-371, et acte V, didascalie initiale, p. 401.

18. "Je trouvais assez bon que Mercure fit la besogne du tentateur. Au bout du compte il faut bien que les dieux soient coupables du mal moral et du mal physique. D'ailleurs Pandore en était plus excusable ; et qu'importe que cette Pandore-Ève soit séduite par Mercure ou par le diable? » (V. à Fériol, $1^{\mathrm{er}}$ avril 1740, D2194)

19. Voir notamment les contributions de L. ANGLIVIEL DE LA BEAUMELLE (« Ève à l'épreuve des Pères », p. 69-87) et F.I. ZEITLIN ("L'Origine de la femme et la femme origine: la Pandore d'Hésiode ", p. 352-355), dans Ève et Pandora. La création de la première femme, éd. par J.-Cl. Schmitt, Paris, Gallimard, 2001.

20. «Ce jour-là, l'ange de l'hymen amena Ève à notre Père dans sa beauté nue, plus ornée, plus charmante que Pandore que les dieux dotèrent de tous leurs dons (oh! trop semblable à elle par le triste événement), alors que conduit par Hermès au fils imprudent de Japhet, elle enlaça l'espèce humaine dans ses beaux regards, afin de venger Jupiter de celui qui avait dérobé le feu 
authentique. » (Le Paradis perdu de Milton, trad. Chateaubriand, Paris, Furne et Gosselin, 1836, p. 289-291.)

21. TROUSSON, Le thème de Prométhée, p. 299.

22. Pandore, acte III, v. 63-140, p. 386-393, et acte IV, v. 1-62, p. 395-398.

23. En supprimant cet épisode qui aurait abordé ouvertement la question des pratiques religieuses, non seulement Voltaire ôte l'idée de vengeance ou de châtiment divin accroissant le sentiment d'injustice mais il se prémunit également contre d'éventuelles critiques de la censure.

24. Pandore, acte I, sc. 2, v. 18, p. 366.

25. Cf. C. GRELL, «La fable au XVIII ${ }^{\mathrm{e}}$ siècle", in Les religions du paganisme antique dans l'Europe chrétienne: XVI ${ }^{e}$-XVIII ${ }^{e}$ siècle, actes du colloque tenu en Sorbonne les 26-27 mai 1987, Paris, PUPS, 1988, p. 81-113.

26. V. à Chabanon, 18 décembre 1767, D14596.

27. Pandore, acte V, v. 43-46, p. 405-406.

28. Pandore, acte I, sc. 2, v. 66-72, p. 370 : «Fuyez, destructeurs odieux / De tout le bien que je veux faire ; / Dieu des malheurs, dieux des forfaits, / Ennemis funèbres, / Replongez-vous dans les ténèbres, / Ennemis funèbres, / Laissez le monde en paix. "

29. Pandore, acte IV, v. 8, p. 383.

30. Pandore, acte V, v. 67 , p. 400.

31. C'est le Destin qui ordonne la paix entre les Titans et les dieux, lesquels doivent « comble[r] cet objet de tous [leurs] dons divers » (acte IV, v. 57, p. 399).

32. Voltaire à Chabanon, 21 décembre 1767, D14607.

33. Pandore, acte II, v. 16-17, p. 374.

34. L'expression est employée aussi bien dans l'article "Épopée » des Questions sur l'Encyclopédie par des amateurs ( $5^{\mathrm{e}}$ partie, [Genève], [Cramer], 1771, p. 228) que dans Pandore où elle est assortie d'une autre expression : « ce feu sacré du tendre amour » (acte II, v. 18, p. 374).

35. Dans la version hésiodique, le feu divin signifie également la vie (cf. VERNANT, Mythe et Société, p. 183). En effet, si le feu n'est encore que le «feu qui cuit », l'œuvre d'Hésiode se situe toutefois «au point même où l'abstrait va se dégager du matériel», explique R. Trousson (Prométhée, p. 39-40).

36. Pandore, acte II, v. 30-32, p. 375.

37. « Vingt-cinquième lettre sur les Pensées de M. Pascal », Paris, Gallimard, 1986, p. 158.

38. Pandore, acte V, v. 162, p. 415.

39. Pandore, acte V, v. 143 et 147, p. 413.

40. Pandore, acte V, v. 148-149, p. 413-414.

41. Samson, éd. R. Goulbourne, in CEuvres complètes de Voltaire, Writing for music 1720-1740, Oxford, Voltaire Foundation, 2008 [ $1^{\text {re }}$ éd. : 1745], vol. 18 C, III, 3, p. 289-290.

42. Cf. TROUSSON, introduction de Pandore, p. 335-342.

43. Pour Voltaire, interprétant Hésiode, la faute originelle qui explique la difficile condition humaine ne damne pas l'homme (V. à Chabanon, 18 décembre 1767, D14596).

44. "L'âme de l'homme est un feu divin que Minerve montre à Prométhée, qui se sert de ce feu divin pour animer l'homme » (art. « Fables » du Dictionnaire philosophique, éd. R. Naves et O. Ferret éd., Paris, Classiques Garnier, 2008 [1 ${ }^{\text {re }}$ éd. :1769], p. 189).

45. Pandore, acte III, v. 73, p. 387.

46. "Il est évident que dans toutes ces promesses et dans toutes ces menaces il n'y a rien que de temporel, et qu'on ne trouve pas un mot sur l'immortalité de l'âme et sur la vie future. [...] Il est très inutile de disputer sur les sentiments secrets de Moïse. Le fait est que dans les lois publiques il n'a jamais parlé d'une vie à venir, qu'il borne tous les châtiments et toutes les récompenses au temps présent » (art. « Âme », Dictionnaire philosophique, p. 15).

47. Pandore, respectivement acte V, v. 155, p. 414, et acte III, v. 117, p. 392. 
48. Cf. GÉLY, L'invention d'un mythe: psyché. Allégorie et fiction, du siècle de Platon au temps de La Fontaine, Paris, Champion, 2006, p. 81.

49. Cette interprétation sera également celle de l'« Apologie de la fable» : «Qu'Hésiode me plaît dans sa théologie / Quand il me peint l'Amour débrouillant le chaos, / S'élançant dans les airs, et planant sur les flots! » (Nouveaux mélanges philosophiques, historiques, critiques, etc., t. III, [s. l. n. n.], 1765, p. 427).

50. «[...] on lut avec beaucoup de plaisir la description du jardin d'Éden, et des amours innocentes d'Adam et d'Ève. En effet, il est à remarquer que dans tous les autres poèmes, l'amour est regardé comme une faiblesse; dans Milton seul il est une vertu. Le poète a su lever d'une main chaste le voile qui couvre ailleurs les plaisirs de cette passion; il transporte le lecteur dans le jardin de délices ; il semble lui faire goûter les voluptés pures dont Adam et Ève sont remplis ; il ne s'élève pas au-dessus de la nature humaine, mais au-dessus de la nature humaine corrompue et comme il n'y a point d'exemple d'un pareil amour, il n'y en a point d'une pareille poésie » (Essai sur la poésie épique, in CEuvres de M. de Voltaire, t. II, [s. 1. n. n.], 1751, p. 91-92).

51. Voir à ce propos l'article «Discours en vers sur l'homme » de D. Acke, dans le Dictionnaire général de Voltaire, R. Trousson et J. Vercruysse éd., Paris, Champion, 2003, p. 360-363.

52. D. SAINTILLAN, « Du festin... », p. 338.

53. Dictionnaire philosophique, art. « Fables », p. 189.

54. Dans Samson déjà, Voltaire empruntait l'expression à Lucrèce (acte IV, sc. 4, v. 49-53, p. 299) : "Amour, volupté pure, / Âme de la nature, / Maître des éléments, / L'univers n'est formé, ne s'anime et ne dure / Que par tes regards bienfaisants. »

\section{AUTEUR}

\section{BÉATRICE FERRIER}

Université d'Artois

« Textes et Cultures » (EA 4028)

beatrice.ferrier@univ-artois.fr fr 